\title{
A experiência das Bibliotecas Virtuais em Saúde nos países de língua portuguesa
}

\author{
The experience of Virtual Health Libraries in Portuguese speaking \\ countries
}

\section{La experiencia de las Bibliotecas Virtuales de Salud en los países de habla portuguesa}

Regina Lucia Ungerer ${ }^{1, a}$

regina.ungerer@gmail.com | http://orcid.org/0000-0002-1218-8827

Paulo Capel Narvai ${ }^{2, b}$

pcnarvai@usp.br | http://orcid.org/o000-0002-4997-9251

\footnotetext{
${ }^{1}$ Fundação Oswaldo Cruz, Centro de Relações Internacionais em Saúde. Rio de Janeiro, RJ, Brasil.

2 Universidade de São Paulo, Faculdade de Saúde Pública. São Paulo, SP, Brasil.

a Mestrado em Saúde da Criança pela Fundação Oswaldo Cruz.

b Doutorado em Saúde Pública pela Universidade de São Paulo.
}

\section{RESUMO}

A Biblioteca Virtual de Saúde dos países de língua portuguesa foi desenvolvida para facilitar o acesso à informação científica e técnica para estudantes, docentes e profissionais de saúde dos países envolvidos. O objetivo deste artigo é descrever a criação e o seu desenvolvimento nos países de língua portuguesa por meio da Rede ePORTUGUÊSe da Organização Mundial de Saúde. Utilizou-se uma metodologia qualitativa baseada em revisão da literatura e análise da documentação técnica, científica e administrativa disponível em repositórios públicos internacionais e instituições nacionais, incluindo relatórios do programa ePORTUGUÊSe entre 2005 e 2015. O desenvolvimento e operacionalização da Biblioteca Virtual de Saúde foi gradual e individualizado e fortemente dependente do envolvimento de profissionais e instituições locais. Mudanças nas prioridades políticas e dificuldades econômicas levaram à sua desaceleração. O aumento da conectividade na África e acesso à Internet estão criando novas oportunidades para retomar seu desenvolvimento nos países de língua portuguesa.

Palavras-chave: Bibliotecas Virtuais em Saúde; Países de língua portuguesa; Capacitação de recursos humanos em saúde; Rede ePORTUGUÊSe; Organização Pan-americana da Saúde; Organização Mundial da Saúde. 


\section{ABSTRACT}

The Virtual Health Library of the Portuguese speaking countries was developed to facilitate access to scientific and technical information for students, teachers, and health professionals in the countries involved. This article aims to describe its development in Portuguese-speaking countries through the World Health Organization's ePORTUGUÊSe Network. It was used a qualitative methodology based on literature review and analysis of technical, scientific, and administrative documentation available in international public repositories and national institutions was used, including reports from the ePORTUGUÊSe program between 2005 and 2015. The development and operation of the Virtual Health Library were gradual and individualized and strongly dependent on the involvement of local professionals and institutions. Changes in political priorities and financial difficulties led their development to slow down. The increase in connectivity in Africa and access to the Internet are creating new opportunities to resume its development in Portuguese-speaking countries.

Keywords: Virtual Health Library; Portuguese-speaking countries; Capacity building of human resources for health; ePORTUGUESe Network; Pan American Health Organization; World Health Organization.

\section{RESUMEN}

La Biblioteca Virtual en Salud de los países de habla portuguesa fue desarrollada para facilitar el acceso a información científica y técnica para estudiantes, profesores y profesionales de la salud en los países involucrados. El objetivo de este artículo es describir el desarrollo de la Biblioteca Virtual en Salud a través de la Red ePORTUGUÊSe de la Organización Mundial de la Salud. Se utilizó una metodología cualitativa basada en la revisión de la literatura y el análisis de la documentación técnica, científica y administrativa disponible en repositorios públicos internacionales e instituciones nacionales, incluidos los informes del programa ePORTUGUÊSe entre 2005 y 2015. El desarrollo y la operacionalización de la BVS fue individualizado y dependió de la participación de profesionales e instituciones locales. Cambios en las prioridades políticas y las dificultades económicas llevaran a su desaceleración. El aumento de la conectividad e Internet en África están creando nuevas oportunidades para reanudar el desarrollo de la Biblioteca Virtual en Salud.

Palabras clave: Biblioteca Virtual de Salud; Países de habla portuguesa; Formación de Recursos Humanos em salud; Red ePORTUGUÊSe; Organización Panamericana de la Salud; Organización Mundial de la Salud.

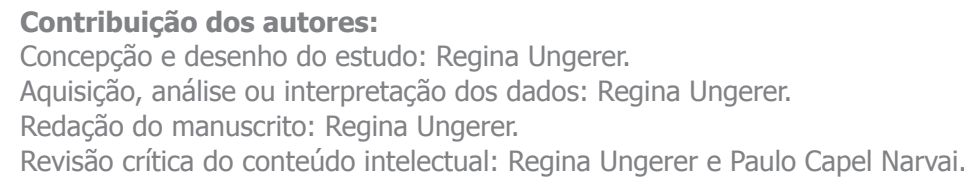

Licença CC BY-NC atribuição não comercial. Com essa licença é permitido acessar, baixar (download), copiar, imprimir, compartilhar, reutilizar e distribuir os artigos, desde que para uso não comercial e com a citação da fonte, conferindo os devidos créditos de autoria e menção à Reciis. Nesses casos, nenhuma permissão é necessária por parte dos autores ou dos editores. 


\section{INTRODUÇÃO}

As Bibliotecas Virtuais em Saúde (BVS) dos países de língua portuguesa foram idealizadas em 2005, logo após a criação da Rede ePORTUGUÊSe da Organização Mundial da Saúde (OMS), com a participação e apoio técnico do Centro Latino Americano e do Caribe de Informação em Ciências da Saúde (BIREME/ OPAS/OMS). Havia uma grande preocupação com a carência de informações de saúde atualizadas nas instituições de ensino superior nos países de língua portuguesa, o que afetava diretamente a formação dos profissionais de saúde.

Durante a Cúpula Ministerial sobre Pesquisa em Saúde de 2004 realizada na cidade do México e promovida pela OMS, Fórum Global de Pesquisa em Saúde e o Governo do México, ficou estabelecido que todos os países e seus parceiros deveriam criar oportunidades para fortalecer ou estabelecer atividades de comunicação para melhorar o acesso e promover o uso de informação em saúde relevante a fim de ajudar os países a cumprirem suas metas estabelecidas nos Objetivos do Desenvolvimento do Milênio'.

O Fórum Global de Pesquisa em Saúde é uma organização internacional independente, criada em 1998 e sediada na Suíça, com o compromisso de destacar a importância da pesquisa, inovação e equidade em saúde. Sua meta era reunir os governos dos países de baixa e média renda, os principais doadores e a comunidade de pesquisa para promover a saúde como um direito, a equidade como princípio e a pesquisa como uma ferramenta indispensável para o desenvolvimento².

Como resposta à essa reunião, a OMS se comprometeu com uma política global e regional de multilinguismo ${ }^{3}$, considerando que grande parte da informação em saúde atualizada e relevante dificilmente alcança os profissionais de saúde nos países em desenvolvimento, especialmente porque circula em idiomas diferentes da língua local.

Apesar desta resposta inicial estar direcionada a aumentar o acesso a publicações nos seis idiomas oficiais das Nações Unidas (inglês, francês, espanhol, russo, chinês e árabe), a cúpula foi também uma oportunidade para criar redes de informação em outros idiomas.

A OMS aproveitou essa oportunidade para atender uma reivindicação dos países de língua portuguesa da África, que, há muito tempo, vinham requisitando a disponibilização de informação em saúde em seu próprio idioma. Neste contexto, foi criada em 2005 a rede ePORTUGUÊSe como uma plataforma para apoiar a colaboração e a troca de informações em saúde entre os países de língua portuguesa no mundo ${ }^{4}$, e que, entre 2005 e 2015, promoveu uma série de iniciativas, disponibilizando diversas ferramentas para facilitar a capacitação de profissionais de saúde nesses países ${ }^{5}$.

Os Estados Membros da OMS de língua portuguesa em 2005 eram: Angola, Cabo Verde, Guiné-Bissau, Moçambique e São Tomé e Príncipe na África, Brasil nas Américas, Portugal na Europa e Timor Leste no Sudeste Asiático. Em 2011, a Guiné Equatorial adotou o português como um de seus idiomas oficiais (juntamente com o francês e o espanhol) e em 2014 passou a fazer parte da Comunidade dos Países de Língua Portuguesa (CPLP), no entanto, a Guiné Equatorial não participou das atividades da Rede ePORTUGUÊSe.

O objetivo inicial desta Rede era contribuir para o desenvolvimento de Bibliotecas Virtuais em Saúde para cada país de língua portuguesa baseado no modelo já desenvolvido com grande sucesso em toda a América Latina e Caribe pela BIREME/OPAS/OMS e, desta forma, apoiar a capacitação de profissionais de saúde.

Contudo, desde o início, ficou clara a enorme potencialidade da Rede ePORTUGUÊSe para fortalecer os sistemas nacionais de saúde, fomentar parcerias e fortalecer a colaboração entre as instituições e profissionais de saúde dos países de língua portuguesa utilizando diversas ferramentas on-line e off-line. A perspectiva é de que seria possível investir no uso das novas tecnologias de informação e comunicação (TIC) para capacitar os recursos humanos e desta forma, diminuir a lacuna entre o saber e o fazer e entre os países desenvolvidos e em desenvolvimento ${ }^{5}$. 
Apesar da baixa conectividade e a intermitência de energia elétrica nos países de língua portuguesa da África, o setor das telecomunicações estava em ascensão e já sendo considerado o de maior crescimento em todos os continentes, com o lançamento de satélites, redes de fibra ótica e cabos submarinos, direcionados a redes de ensino, universidades e órgãos governamentais. No ano 2000, o número de usuários da Internet na África era 4,5 milhões, em 2010 os usuários eram 100 milhões, e em 2018 já eram mais de 450 milhões ou $35,2 \%$ da população total 6 .

Neste contexto propício foi criada a Rede ePORTUGUÊSe para promover maior intercâmbio de informação em saúde junto aos Estados Membros da OMS de língua portuguesa com a finalidade de fortalecer a colaboração entre as instituições e profissionais de saúde destes países compartilhando recursos e informação em saúde em português.

Este artigo tem o objetivo de rever o processo de criação e desenvolvimento das Bibliotecas Virtuais em Saúde Nacionais (BVS) nos países de língua portuguesa por meio da análise de documentos selecionados dentre os produzidos, coletados e armazenados pela Rede ePORTUGUÊSe da OMS no período de 2005 a 2015; além de identificar novas oportunidades de cooperação entre os países de língua portuguesa para dar continuidade ao desenvolvimento das BVS em seus países.

\section{ABORDAGEM METODOLÓGICA}

Este foi um estudo de caráter qualitativo que teve como objetivo descrever e analisar o processo de criação das Bibliotecas Virtuais em Saúde Nacionais nos países de língua portuguesa e seu desenvolvimento no período de 2005 a 2015.

Foi realizada uma revisão bibliográfica e análise da documentação técnica, cientifica e administrativa disponível sobre o desenvolvimento das BVS nos países de língua portuguesa encontradas principalmente na plataforma da BVS ePORTUGUÊSe, disponível em http://www.bireme.br/php/index.php. Este é um espaço virtual de acesso livre para coleção de fontes de informação em saúde desenvolvida pela BIREME/ OPAS/OMS. A documentação especifica da BVS Nacional de cada país analisada está disponível em:

- Angola: http://angola.eportuguese.org

- Brasil: http://brasil.bvs.br

- Cabo Verde: http://cabo-verde.eportuguese.org

- Guiné-Bissau: http://guine-bissau.eportuguese.org

- Moçambique: http://mocambique.eportuguese.org

- Portugal: http://portugal.eportuguese.org

- São Tomé e Príncipe: http://sao-tome-principe.eportuguese.org

- Timor Leste: http://timor-leste.eportuguese.org

Além disso, foram identificados e analisados outros documentos disponíveis nos países relacionados e armazenados na coordenação do programa ePORTUGUÊSe da OMS e no Espaço Colaborativo ePORTUGUÊSe (http://cspace.eportuguese.org/).

\section{CRIAÇÃO E IMPLEMENTAÇÃO DAS BVS NOS PAÍSES DE LÍNGUA PORTUGUESA}

Em 2004, respondendo ao compromisso de melhorar o acesso à informação em saúde para todos os seus Estados Membros, a OMS apresentou uma proposta para a criação de redes eletrônicas especificas (eHealth Networks) para apoiar comunidades de conhecimento regionais tais como as já existentes em 
árabe na Região do Mediterrâneo Oriental (EMRO) e em espanhol na Região das Américas (AMRO). Como proposta inicial, o foco seria nos Estados Membros da OMS de língua portuguesa, mas que posteriormente, poderia ser expandido para outras regiões ou grupo de países, como, por exemplo: a Federação Russa e os países do antigo bloco soviético. Na prática, nenhuma outra rede eletrônica baseada em idioma foi desenvolvida pela OMS.

Alguns fatores contribuíram para que esta rede eletrônica começasse com o idioma português. Primeiro, os países de língua portuguesa, especialmente Angola e Moçambique, reivindicavam à OMS informação de saúde atualizada, relevante e baseada na melhor evidência disponível em seu idioma para melhorar o atendimento de sua população; segundo, havia um país de língua portuguesa em quatro continentes e quatro regiões da OMS, (África subsaariana - AFRO; Américas - AMRO; Europa - EURO e Sudeste Asiático - SEARO); terceiro, em 2004, a maioria dos países de língua portuguesa estavam classificados, de acordo com o $\mathrm{PNUD}^{7}$ como sendo de baixo ou médio desenvolvimento; e quarto, já havia interesse por parte do Centro Latino Americano e do Caribe de Informação em Ciências da Saúde (BIREME/OPAS/OMS) em desenvolver o modelo da BVS em Moçambique ${ }^{8}$, e sua interface já existia em espanhol, inglês e português.

Havia também uma grande preocupação com a carência de informações atualizadas nas instituições de ensino superior dos países de língua portuguesa que afetava diretamente a formação dos profissionais de saúde. Nesse mesmo sentido, um estudo de 2003 ressaltava as dificuldades dos profissionais da informação em saúde na África que poderiam ser resumidas em duas palavras: treinamento e acesso9.

O objetivo de desenvolver o modelo da BVS nos países de língua portuguesa foi fortemente embasado na Resolução 58/28 da Assembleia Mundial da Saúde de 2005. Ela enfatizava o uso custo-efetivo e seguro de tecnologias de informação e comunicação no campo da saúde, inclusive para o atendimento e prestação de serviços, vigilância, educação em saúde e pesquisa, e recomendava aos Estados Membros que estabelecessem redes e centros nacionais de excelência na área de eHealth para o desenvolvimento de melhores práticas, políticas de coordenação e apoio técnico para a prestação de serviços, melhoria do atendimento, informação ao público, capacitação e vigilância ${ }^{10}$.

Com esta resolução criaram-se oportunidades para fomentar novas parcerias e fortalecer a colaboração entre instituições e profissionais de saúde dos países de língua portuguesa. O primeiro passo em direção à construção da BVS nestes países foi a identificação de profissionais que serviriam de elo entre a Rede ePORTUGUÊSe e os respectivos Ministérios da Saúde. Quando selecionados, estes pontos focais foram convidados a participar do $7^{\circ}$ Congresso Latino-Americano e do Caribe de Informação em Ciências da Saúde (CRICS7) e da $4^{\text {a }}$ Reunião de Coordenação Regional da Biblioteca Virtual em Saúde, promovidos pela OPAS/OMS e realizado em Salvador da Bahia, Brasil, em setembro de $2005^{11}$. Essa foi a primeira vez que representantes dos países africanos de língua oficial portuguesa (PALOP) foram convidados a participar deste evento Latino-Americano, sendo uma oportunidade para aprenderem sobre o modelo da BVS e apresentarem as condições de acesso e gestão da informação em saúde em seus países. Ao final, ficou claro que havia grandes diferenças entre os países, e a grande expectativa entre os participantes era que o desenvolvimento das BVS respeitasse as individualidades de cada local e que sua implantação fosse gradual. Três países relataram a inexistência de bibliotecas da saúde e, praticamente, ausência de material em português nas Bibliotecas dos Escritórios de Representação da OMS local, dificultando muito a pesquisa dos profissionais de saúde que utilizavam estas bibliotecas para se atualizar. Além disso, a produção de informação técnico e científica era muito baixa, e somente Moçambique produzia uma revista médica. Devido à dificuldade de encontrar informação atualizada, os profissionais de saúde não tinham o hábito de frequentar bibliotecas ou centros de documentação, esta, portanto, seria uma oportunidade de mudar a cultura existente.

Na sequência, foi realizado em 2007, o primeiro seminário das Bibliotecas Virtuais em Saúde nos países de língua portuguesa na sede da BIREME/OPAS/OMS em São Paulo com a colaboração técnica do 
Ministério da Saúde do Brasil e da OMS. O tema central foi 'Como melhorar o acesso à informação científica e técnica em saúde nos países de língua portuguesa - O Modelo da Biblioteca Virtual em Saúde'. No encontro, representantes de seis países de língua portuguesa se comprometeram a iniciar a discussão e o desenvolvimento da BVS em seus países, envolvendo outras instituições de ensino e pesquisa para a criação das instâncias da BVS, como o comitê executivo, o comitê consultivo e a secretaria executiva, seguindo o modelo da matriz de responsabilidades definido pela BIREME/OPAS/OMS para as Bibliotecas Virtuais em Saúde.

O documento norteador produzido nesse encontro tinha três objetivos principais:

1. Cada BVS deveria ser adaptada às condições locais, incluindo sua vinculação aos planos e sistemas nacionais de saúde, considerando as dimensões de promoção e atenção à saúde, educação e formação de recursos humanos e de pesquisa.

2. As BVS deveriam servir para incentivar o compartilhamento e o intercâmbio das coleções nacionais de produtos, serviços e eventos.

3. As BVS deveriam servir como bases de interação com redes regionais e internacionais ${ }^{12-13}$.

Na figura 1, encontra-se o registro desta reunião.

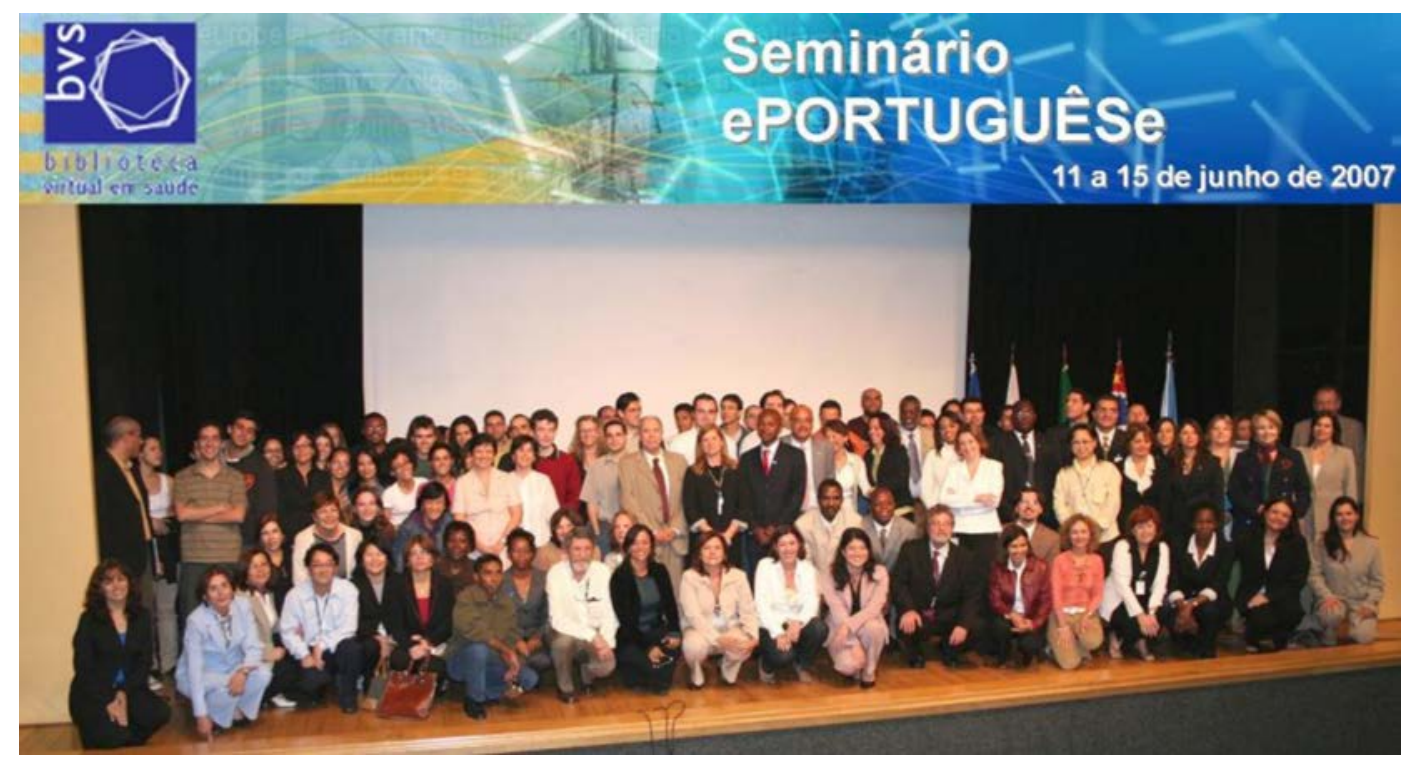

Figura 1 - Foto de família no início do seminário "Como melhorar o acesso à informação científica e técnica em saúde nos países de língua portuguesa - O Modelo da Biblioteca Virtual em Saúde" com os representantes de todos os países de língua portuguesa, Ministério da Saúde do Brasil e BIREME/OPAS/OMS Fonte: Acervo da autora.

A partir deste seminário, desencadeou-se o processo de capacitação dos profissionais que seriam os responsáveis por desenvolver e avançar com as BVS Nacionais em seus países.

Em 2008, com um financiamento específico para São Tomé e Príncipe, um profissional de saúde, um profissional da informação e um bibliotecário desse país foram os primeiros a receber treinamento para o desenvolvimento de sua BVS Nacional na sede da BIREME/OPAS/OMS ${ }^{14-15}$. No mesmo ano, dois profissionais da Guiné-Bissau foram convidados a participar do $8^{\circ}$ Congresso Latino-Americano e do Caribe de Informação em Ciências da Saúde (CRICS8) no Rio de Janeiro e, em seguida, foram treinados na sede da BIREME/OPAS/OMS para o desenvolvimento de sua BVS Nacional. Estes dois países foram os primeiros a criar as páginas de rosto de suas Bibliotecas Virtuais em Saúde e, de volta a seus países, tinham o compromisso de se reunir com outras instituições e profissionais de diversas áreas para dar seguimento à $\mathrm{BVS}^{16}$. 
Em 2009, foi a vez dos profissionais de Angola, Cabo Verde, Moçambique e Timor Leste receberem o mesmo treinamento na sede da BIREME/OPAS/OMS para o desenvolvimento de suas respectivas BVS Nacionais $^{17-18}$. Nesta oportunidade foi lançado o portal da BVS da Rede ePORTUGUÊSe, que seria a plataforma de entrada para todas as BVS Nacionais. Todos retornaram aos seus países com as páginas de suas BVS criadas para dar continuidade ao seu processo de desenvolvimento.

A BVS Portugal começou a ser desenvolvida em colaboração com a Fundação para a Computação Científica Nacional em 2009. Em 2011, Portugal apresentou a sua BVS Nacional durante a III Reunião de Coordenação da BVS da Rede ePORTUGUÊSe, em São Tomé e Príncipe, completando o quadro dos oito países de língua portuguesa com suas BVS Nacionais desenvolvidas dentro da mesma plataforma.

No Brasil, o desenvolvimento das BVS começou em 1999 com um acordo de cooperação entre a Organização Pan-Americana da Saúde (OPAS) e o Ministério da Saúde do Brasil para desenvolver uma BVS sobre Saúde Pública e uma BVS sobre saúde do adolescente. Este acordo de cooperação impulsionou uma série de ações que foram fundamentais para o crescimento da BVS no Brasil, já que o modelo seguia os padrões internacionais de operacionalização, facilitando o acesso livre e gratuito a bases de dados nacionais e internacionais ${ }^{19}$. Na figura 2, encontra-se a página de rosto da BVS da Rede ePORTUGUÊSe, com acesso a todas as BVS nacionais de todos os países.

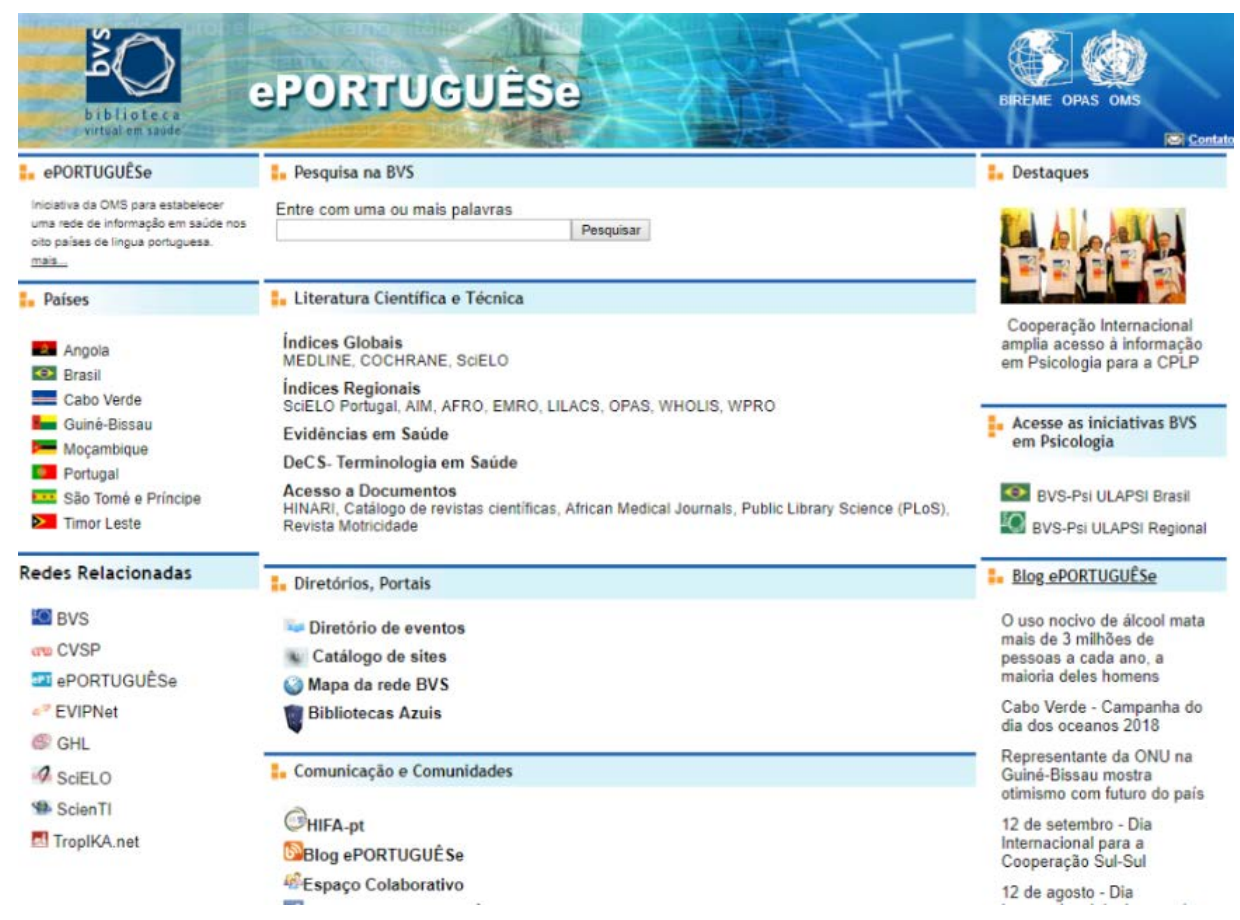

Figura 2 - BVS da Rede ePORTUGUÊSe desenvolvida no modelo da BIREME/OPAS/OMS. Porta de entrada para as BVS dos países de língua portuguesa

Fonte: Página de rosto da BVS da Rede ePORTUGUÊSe (http://www.bvs.eportuguese.org)

Apesar das Bibliotecas Virtuais em Saúde nacionais terem sido desenvolvidas na mesma plataforma e, portanto, com características similares em suas páginas de rosto, elas também apresentavam singularidades construídas de acordo com os interesses e valores dos pontos focais responsáveis por sua criação, por exemplo, as fontes de dados específicas, literatura científica regional ou documentos de interesse para o país. Destacavam também o uso da tecnologia RSS (Really Simple Syndication), que permite inscrever sites de internet que atualizam seus conteúdos regularmente. Assim a BVS de Angola, da Guiné Bissau, de São Tomé e Príncipe e de Timor Leste tinham seus "feeds RSS" no Blog ePORTUGUÊSe; Cabo Verde atualizava as notícias do Ministério da Saúde; Moçambique destacava as notícias do Instituto Nacional de Saúde (INS); e Portugal destacava as notícias publicadas no Espaço Colaborativo. Além disso, os cabeçalhos 
de cada país representavam paisagens características, acontecimentos locais, itens típicos e algumas personalidades importantes, dando a cada BVS uma marca individual.

A vantagem desse modelo de Bibliotecas Virtuais em Saúde é que podiam (e podem) ser acessadas de qualquer lugar, a partir de uma conexão de internet, bastando apenas um local físico para a instalação dos servidores de armazenamento dos dados e dos computadores para o acesso dos usuários. Em Angola, a BVS foi alocada na Escola Nacional de Saúde Pública (ENSPA); em Cabo Verde, a BVS ficou a cargo do Centro Nacional de Desenvolvimento Sanitário (CNDS). Em 2014, o CNDS passou a ser chamado de Instituto Nacional de Saúde Pública (INSP), complementando o trabalho da Direção Nacional da Saúde, com destaque para a vigilância em saúde de Cabo Verde. Na Guiné-Bissau, o Instituto Nacional de Saúde (INASA) foi o local escolhido para abrigar a BVS; em Moçambique, foi o Centro de Documentação do Instituto Nacional de Saúde (INS); em São Tomé e Príncipe, a Biblioteca Nacional disponibilizou uma sala para ser usada como biblioteca da saúde com um local para leitura e uma sala para os serviços administrativos; em Timor Leste, a BVS ficou no Instituto de Ciências da Saúde (ICS).

Todos os PALOP receberam por meio da Rede ePORTUGUÊSe computadores, servidores, impressoras entre outros equipamentos para dar início ao processo de desenvolvimento das BVS Nacionais. O Alto Comissariado da Saúde de Portugal foi o responsável pela criação da BVS Portugal, e sendo um país de mais alto desenvolvimento, não recebeu equipamentos eletrônicos. No Brasil, a BVS saúde pública Brasil (BVSSP Brasil) foi a primeira a ser desenvolvida em 1999 em parceria com o Ministério da Saúde, Organização Pan Americana da Saúde (OPAS), Faculdade de Saúde Pública da Universidade de São Paulo (FSP-USP), Instituto de Comunicação e Informação Científica e Tecnológica em Saúde (Icict/Fiocruz) e a Fundação Oswaldo Cruz (Fiocruz). A instituição responsável pela coordenação da BVS-SP Brasil é a biblioteca de saúde pública da Fiocruz. Essa BVS, além dos serviços tradicionais, também é um espaço que disponibiliza um programa de capacitação de produtores, intermediários e usuários para sua operação por meio de cursos presenciais ou a distância. Ainda em 1999, a Área da Saúde do Adolescente e do Jovem do Ministério da Saúde do Brasil desenvolveu uma parceria com a BIREME/OPAS/OMS para criar uma BVS sobre a saúde do adolescente (BVS Adolec) com o objetivo de motivar a discussão, em rede, de profissionais da área.

Como forma de incentivar a troca de informações e conhecimentos sobre o desenvolvimento de cada BVS Nacional, foi desenvolvido um espaço colaborativo para que a informação disponível fosse construída de forma coletiva com a participação de todos os seus membros.

O desenvolvimento deste espaço colaborativo atendeu ao pedido dos diretores de recursos humanos para a saúde dos PALOP durante o primeiro encontro da Cooperação Técnica da Comissão Europeia, Organização Mundial da Saúde e Países africanos de língua oficial portuguesa (CE/OMS/PALOP) em apoio ao desenvolvimento de recursos humanos em saúde realizado em dezembro de 2008 em Cabo Verde ${ }^{20}$.

O espaço colaborativo ePORTUGUÊSe, cujo site é mantido até hoje pela BIREME/OPAS/OMS, pode ser acessado no link (http://cspace.eportuguese.org) e tornou-se um local preferencial para compartilhar notícias, documentos, fotos, disseminar campanhas de saúde etc. em cada país. Sua interface simplificada permitia que notícias pudessem ser inseridas por qualquer membro registrado na Rede, tornando-se, consequentemente, um instrumento interativo e bastante utilizado. O espaço colaborativo publicou mais de 2100 notícias de todos os países de língua portuguesa, além de 45 coleções de fotos e 52 arquivos de documentos relacionados à BVS e a Rede ePORTUGUÊSe que continuam disponíveis em seu site.

Considerando as prioridades de cada país e avaliando as parcerias existentes, quer sejam formais ou informais, entre instituições locais de ensino e pesquisa dos países de língua portuguesa e instituições e universidades brasileiras e portuguesas, esses países têm a vantagem de estar conectados pelo idioma e de fazer parte da Comunidade dos Países de Língua Portuguesa (CPLP). Muitos profissionais desta comunidade estão acostumados a trocar informações de saúde, seja individualmente seja por meio de suas instituições, com outras redes de informação já existentes, como, por exemplo, o fórum de discussão HIFA-pt (health 
information for all em português). Este fórum foi criado em 2009 com o apoio da Rede ePORTUGUÊSe visando a incluir os profissionais de saúde, formuladores de políticas, gestores, bibliotecários e profissionais da informação dos países de língua portuguesa em um grupo de discussão amplo e baseado na rede global de saúde Health Information for all (https://www.hifa.org/). Tal grupo era baseado no Reino Unido, com mais de 18.000 membros comprometidos com o objetivo de fazer com que todas as pessoas possam acessar as informações de saúde que necessitam.

Entre os diversos benefícios das BVS para as instituições e os profissionais de saúde, destacava-se a possibilidade de estudantes, docentes e pesquisadores terem, à sua disposição, uma literatura de referência em português, informação científica internacional, acesso à documentação nacional em saúde, e de permitir o intercâmbio entre os profissionais de saúde dos vários países, possibilitando o desenvolvimento de competências profissionais individuais com responsabilidade e segurança.

Esperava-se que o uso das BVS pudesse fomentar uma cultura de pesquisa científica e tomada de decisão baseada em evidência no seio dos profissionais, nos diversos níveis dos sistemas de saúde.

\section{EVOLUÇÃO DAS BVS NOS PAÍSES DE LÍNGUA PORTUGUESA}

Por sua diversidade e por encontrar diferenças significativas entre os países, o desenvolvimento das BVS foi desigual em cada lugar e dependeu da capacidade local de se apropriar e implementar as atividades propostas levando em consideração suas diferenças culturais e socioeconômicas.

Moçambique foi o país que mais evoluiu no desenvolvimento de sua BVS Nacional. Conseguiu negociar com o Ministério da Saúde (MINSA) o estabelecimento de políticas e programas nacionais para reforçar a capacidade de gestão e operacionalização em prol do acesso equitativo à informação e evidência científica em saúde. Isso resultou na criação de um espaço dentro da Biblioteca Nacional de Saúde para o uso da BVS e a constituição dos Comitês Consultivo, Executivo e secretaria executiva para a gestão da BVS Moçambique.

A BVS Cabo Verde fez parte das iniciativas do Governo e do portal científico inaugurado em 2012 pelo Ministério do Ensino Superior e Ciências e continua interligada com outras bibliotecas nacionais. O Comitê Consultivo da BVS Cabo Verde foi constituído em 2011 com representantes de diversos setores, tais como a ordem dos médicos, associação dos enfermeiros, Ministério da Educação, Universidades, Biblioteca Nacional, Núcleo Operacional da Sociedade de Informação (NOSI), entre outros.

A BVS Guiné Bissau formalizou a criação de seu Comitê Consultivo em 2011, com representantes de oito instituições de diversos setores, mas ficaram aguardando a aprovação formal do Conselho de Ministros e, com o passar do tempo, não avançaram para além disso.

São Tomé e Príncipe conseguiu organizar três seminários internos de formação sobre os métodos de utilização da BVS destinados a capacitar bibliotecários, profissionais e técnicos de saúde no manejo correto da busca da informação para melhoria de suas atividades profissionais. Em 2010, por despacho do Ministro da Saúde, o país oficializou a criação da BVS Nacional e definiu as instituições que deveriam fazer parte dos comitês consultivo, executivo e secretaria executiva. No entanto, não avançaram mais apesar de terem acolhido a III Reunião de Coordenação das BVS da Rede ePORTUGUÊSe em 2011.

Para a BVS Portugal, foi assinado um protocolo de colaboração com a Fundação para a Computação Científica Nacional com o objetivo de desenvolver e implementar sua BVS e constituir seu Comitê Consultivo com representantes da Direção Geral de Saúde, área acadêmica, investigação etc., mas não evoluiu muito além. Timor Leste promoveu reuniões com profissionais e outras instituições na tentativa de criar o comitê consultivo para a operacionalização de sua BVS, mas também não avançou.

No Brasil, já foram criadas 19 BVS temáticas nas mais diversas áreas, sendo que 15 continuam ativas e quatro necessitam de atualização. Foram desenvolvidas duas BVS biográficas sobre Adolpho Lutz e Carlos Chagas e cinco BVS institucionais, a saber: Ministério da Saúde, Fiocruz, Instituto Evandro Chagas, 
Secretaria Estadual de Saúde de São Paulo e Secretaria Municipal de Saúde de São Paulo. Todas as BVS no Brasil são parte integrante da Biblioteca Virtual em Saúde para a América Latina e Caribe.

Angola não evoluiu para além da criação da página de rosto de sua BVS.

Havia a expectativa de que os países identificassem os recursos necessários para elaborar planos específicos a médio e longo prazo a fim de desenvolver atividades de promoção da BVS, e, dessa forma, garantir a continuidade do seu desenvolvimento. Porém, não foi possível elaborar ferramentas de apoio ou matriz de responsabilidades para a gestão da BVS localmente, especialmente pelas dificuldades encontradas pelos pontos focais em disseminar e divulgar a BVS dentro do país, pela grande rotatividade dos responsáveis em busca de melhor remuneração ou mesmo término de contrato laboral, mas também pelo pouco envolvimento dos gestores nos Ministérios da Saúde de cada país.

Com o passar do tempo e mudanças de governos, os pontos focais foram sendo substituídos, e as políticas e prioridades dos diferentes Ministérios da Saúde também foram modificadas. Além disso, a crise financeira de 2008 comprometeu ações de cooperação internacional, e os recursos financeiros para promover capacitações locais tornaram-se mais escassos. Isso contribuiu para o fraco envolvimento de profissionais e instituições de ensino e pesquisa no crescimento e disseminação de suas BVS nacionais.

Com o término da Rede ePORTUGUÊSe em 2015, desfizeram-se parcerias nacionais e internacionais, e foram encerradas as atividades de cooperação no campo. Foram também desfeitas as atividades de monitoramento e acompanhamento das ações que pudessem incentivar os profissionais de saúde, alunos e docentes a utilizarem a BVS e, dessa forma, diminuir seu isolamento profissional.

Além disso, a baixa conectividade, o restrito acesso à Internet e o fornecimento irregular de energia elétrica, em alguns países, foram fatores que contribuíram para que as BVS não tivessem o desenvolvimento esperado. A tabela 1 mostra o acesso à internet nos países de língua portuguesa nos anos de 2010 e 2019.

\section{Tabela 1-Acesso à internet nos países de língua portuguesa, 2010 e 2019}

\begin{tabular}{lcc} 
País & $\begin{array}{c}\text { Acesso à Internet } \\
\mathbf{2 0 1 0}\end{array}$ & $\begin{array}{c}\text { Acesso à Internet } \\
\mathbf{2 0 1 9}\end{array}$ \\
\hline Angola & $4,3 \%$ & $22,3 \%$ \\
Brasil & $34 \%$ & $70,7 \%$ \\
Cabo Verde & $23,9 \%$ & $62,8 \%$ \\
Guiné-Bissau & $2,4 \%$ & $7,7 \%$ \\
Moçambique & $1,6 \%$ & $20,8 \%$ \\
Portugal & $41,8 \%$ & $78,2 \%$ \\
São Tomé e Príncipe & $11,2 \%$ & $29,9 \%$ \\
Timor Leste & $0,2 \%$ & $30,3 \%$ \\
\hline
\end{tabular}

Fonte: Elaboração própria (2019).

No entanto, as Bibliotecas Virtuais em Saúde (BVS) continuam sendo uma ferramenta importante para os profissionais de saúde dos países de língua portuguesa. Atualmente, não há informação sobre a existência de profissionais ou instituições especializadas em gestão de conhecimento ou em tecnologias de informação e comunicação que possam atuar, desenvolver ou manter as BVS. Tampouco há informações sobre reuniões dos comitês consultivos que atuem nas tomadas de decisões, definição de prioridades ou estabelecimento de um plano de trabalho para dar seguimento às BVS. Apesar de não haver atividade no desenvolvimento das Bibliotecas Virtuais em Saúde Nacionais, elas permanecem on-line, sendo mantidas nos servidores da 
BIREME/OPAS/OMS. À exceção da BVS Brasil, nenhuma outra BVS tem sido atualizada, apesar de ter havido algum interesse de Cabo Verde e Moçambique para dar continuidade às suas BVS.

\section{CONSIDERAÇÕES FINAIS}

São inegáveis as potenciais implicações das TIC e da Internet em particular como facilitador de recursos educacionais e de laboratórios virtuais para a formação de profissionais em qualquer país e especialmente na África Subsaariana, onde as instituições de ensino enfrentam desafios técnicos, financeiros e de infraestrutura importantes.

A possibilidade de alcançar profissionais em qualquer lugar e, assim, disseminar conteúdos como artigos, livros e documentos, de forma gratuita, não pode ser menosprezada. De fato, as Bibliotecas Virtuais em Saúde dos países de língua portuguesa foram importantes para levar a informação em saúde atualizada relevante aos oito Estados Membros da OMS de língua portuguesa à época.

Porém, o fim da Rede ePORTUGUÊSe contribuiu para a dispersão das atividades conjuntas e, sem o apoio da OMS, os países passaram a ter o desafio de garantir o desenvolvimento e manutenção das Bibliotecas Virtuais em Saúde e suas redes de cooperação individualmente e como ainda não eram muito conhecidas, suas potencialidades não foram mais exploradas. Além disso, a escassez, e mesmo ausência, de recursos financeiros nos países para a realização de atividades descentralizadas contribuiu para a inativação das BVS Nacionais.

Com a melhoria da conectividade e maior acesso à Internet nos países de língua portuguesa, voltou a crescer também o interesse em atividades de médio e longo prazo que pudessem beneficiar a capacitação sustentável de Recursos Humanos para a Saúde (RHS), a disseminação da informação para outros profissionais e outras instituições de saúde, especialmente nas zonas rurais e distantes dos centros urbanos.

Abre-se, assim, a possibilidade de retomar o desenvolvimento das Bibliotecas Virtuais em Saúde nos países de língua portuguesa. Porém, essas bibliotecas não serão reativadas sozinhas; na prática, isso significa que será também necessário intensificar a discussão de estratégias de disseminação e apropriação das BVS pelos países de língua portuguesa para que estas possam ser melhor aproveitadas. Assim como será necessário resgatar as redes de instituições científicas locais e impulsioná-las para a fase seguinte da BVS. Para dar visibilidade e apoio à produção local de conhecimento, será importante o compromisso de pontos focais e de profissionais de saúde, assim como o empenho das instituições para dar continuidade à rede BVS.

Deve-se também ressaltar a importância de se criar uma política de sensibilização de gestores e tomadores de decisão sobre o conceito da BVS e o acesso à informação em saúde para formuladores de políticas, estudantes e docentes como uma alternativa de aprendizado dentro do setor da saúde e da educação em saúde. Portanto, é de grande relevância refletir sobre o processo de consolidação das BVS e os benefícios que elas, com certeza, trarão para os profissionais de saúde e instituições de ensino dos países de língua portuguesa.

O diálogo e a discussão compartilhada entre profissionais de saúde que vivem em países diferentes enfrentando realidades distintas são muito enriquecedores e ilustram bem as vantagens que a conectividade e as TIC podem trazer para o dia a dia dos profissionais de saúde.

No entanto, não basta a aquisição de equipamentos eletrônicos ou incentivar o uso das BVS para o acesso à informação, é preciso também incorporar mudanças firmes no processo de aprendizagem e uso das TIC para a capacitação e treinamento de profissionais de saúde para que estes sintam-se confiantes para trabalhar com um novo conceito de rede que favoreça a cooperação transversal e fortaleça parcerias individuais e institucionais. Ou seja, será necessário planejamento e articulação entre os parceiros de todos os países. 
Decerto, a BIREME/OPAS/OMS foi a instituição responsável pelo desenvolvimento e manutenção de capacidades para a gestão da BVS e a sua implantação nos países. Hospeda, até hoje, o portal da BVS ePORTUGUÊSe, o Espaço Colaborativo e cada uma das oito instâncias das BVS nacionais e suas fontes de informação, apesar de seus planos de desenvolvimento estarem paralisados e sem receber nenhuma contribuição local, já que os profissionais que haviam sido treinados para mantê-las não mais realizam esta função.

Hoje, com mais de dez anos de experiência e conhecendo melhor as realidades locais, é certo que um dos grandes desafios dos países de língua portuguesa foi (e ainda é) a ausência de instituições nacionais produtoras de conhecimento científico em saúde com profissionais capacitados para operar as metodologias necessárias para manter e atualizar as BVS Nacionais. Não havia como desenvolver o modelo proposto com todas as suas instâncias.

Em outras palavras, qualquer ação, programa ou plano de cooperação construído em "bloco" contemplando diversos países com graus de desenvolvimento diversos, com suas próprias crenças, línguas, culturas e com sistemas de saúde diferentes, deveria ter considerado as dificuldades na sua implantação. Conhecendo hoje as necessidades reais das instituições de saúde e ensino em cada país, talvez seja mais fácil propor ações para captação de fundos a fim de garantir que o acesso à informação relevante e atualizada em seu próprio idioma possa ser levado a todos.

Neste sentido, nos parece que a melhor estratégia para reativar as BVS dos países de língua portuguesa seria a reativação da BVS ePORTUGUÊSe como uma única biblioteca com sub-coleções para cada um dos PALOP e Timor Leste, fortalecendo o trabalho colaborativo e em rede. Como a BIREME/OPAS/ OMS continua disponível para avançar com o desenvolvimento das ações de gestão da informação e do conhecimento, bastaria recriar as parcerias com instituições locais interessadas nos países.

O mesmo poderia ser feito com o Espaço Colaborativo (ECOL), criado para manter a discussão sobre o desenvolvimento das BVS, que continua a ser mantido pela BIREME/OPAS/OMS. Neste caso, a ferramenta utilizada para o ECOL está desatualizada e foi descontinuada, o que exigiria o uso de outra metodologia.

\section{REFERÊNCIAS}

1. World Health Organization. Report from the Ministerial Summit on Health Research: identify challenges, inform actions, correct inequities [Internet]. 2005 [citado em 2017 ago 30]. Disponível em: https:// apps.who.int/iris/handle/10665/43226.

2. Global Forum for Health Research [Internet]. 2017 [citado em 2017 ago 30]. Disponível em: http:// www.who.int/workforcealliance/members partners/member list/gfhr/en/.

3. World Health Organization. Multilingualism: implementation of Action Plan. [Internet]. In: $71^{\circ}$ World Health Assembly. Geneva; 2008 abr. [citado em 2018 ago 23]. Disponível em: http://www.who.int/ eportuguese/mission/6131.pdf?ua=1.

4. Ungerer RLS. Rede ePORTUGUÊSe: uma nova ferramenta para os países de língua portuguesa. In: Mathias I, Monteiro A. Gold book: inovação tecnológica em educação e saúde [Internet]. Rio de Janeiro: EdUER], 2012 [citado em 2020 jul 20]; p.487-500. Disponível em: http://www.telessaude.uerj.br/ goldbook. ISBN 9788575112380.

5. Ungerer RLS. The ePORTUGUESe network experience. UN Special [Internet]. 2014 jan [citado em 2017 ago 25]:16-17. Disponível em: https://www.unspecial.org/wpcontent/ uploads/2014/01/UNSpecial Janvier2014.pdf.

6. Internet World Stats [Internet]. 2019 [citado em 2019 set 12]. Disponível em: https://www. internetworldstats.com/africa.htm.

7. PNUD. Programa das Nações Unidas para o Desenvolvimento. Relatório do Desenvolvimento Humano [Internet]. 2004 [citado em 2018 set 05. Disponível em: https://www.br.undp.org/content/brazil/pt/home/ library/idh/relatorios-de-desenvolvimento-humano/relatorio-do-desenvolvimento-humano-20004.html. 
8. $\quad$ Centro Latino-Americano e do Caribe de Informação em Ciências da Saúde. Project Proposal for introducing Mozambique into the Virtual Health Library [Internet]. 2005 [citado em 2018 set 05]. Disponível em: http://espacio.bvsalud.org/boletim.php?newsletter $=20050727 \&$ newsLang $=$ pt\&newsName=Newsletter\%20BVS\%20011\%2027/julho/2005\&articleId =07112626200520.

9. Bob I. International Funding Priorities for Health Information. Hypothesis [Internet]. 2003 [citado em 2018 ago 23];17:1. Disponível em: https://www.mlanet.org/d/do/837.

10. World Health Organization. Resolução WHA58/28 - eHealth [Internet]. In: $58^{\circ}$ World Health Assembly. Geneva; 2005 maio [citado em 2018 ago 23]. Disponível em: https://www.who.int/healthacademy/ media/WHA58-28-en.pdf.

11. Newsletter BVS 011 27/julho/2005. [citado em 2018 set 05]. Disponível em: http://espacio.bvsalud.org/ boletim.php?newsletter $=20050727$ \&newsLang $=$ pt\&newsName $=$ Newsletter\%20BVS\%20011\%2027/julho L2005\&articleId=07112626200520.

12. Espaço Colaborativo ePORTUGUÊSe. I Reunião de Coordenação da Rede ePORTUGUÊSe: Como melhorar o acesso a informação técnico e científica em saúde nos países de língua portuguesa e o modelo da BVS [Internet]. 2007 [citado em 2020 jul 20]. Disponível em: http://cspace.eportuguese.org/tikilist file gallery.php? galleryId $=2$.

13. Centro Latino-Americano e do Caribe de Informação em Ciências da Saúde. Como melhorar o acesso à informação científica e técnica em saúde nos países de língua portuguesa - O Modelo da Biblioteca Virtual em Saúde (BVS) [Internet]. 2007 [citado em 2020 jul 20]. Disponível em: http://cspace. eportuguese.org/tiki-download file.php?fileId $=1292$.

14. Ungerer R. Projeto para a implementação e desenvolvimento de uma Biblioteca Virtual em Saúde (BVS) em São Tomé \& Príncipe baseada no modelo desenvolvido pelo Centro Latino Americano e do Caribe de informação em ciências da saúde (BIREME). Relatório técnico. Genebra; 2008.

15. Espaço Colaborativo ePORTUGUÊSe. 2 a 13 de junho: capacitação do modelo BVS para São Tomé e Príncipe [Internet]. 2008 [citado em 2020 jul 20]. Disponível em: http://cspace.eportuguese.org/tikiread article.php?articleId $=5$.

16. Ungerer R. Development of a Health Information Network - Guinea Bissau and Mozambique. Progress Report (Project 56/5). Genebra; 2009.

17. Espaço Colaborativo ePORTUGUÊSe. Capacitação para Angola, Cabo Verde, Moçambique e Timor Leste [Internet]. 2009 [citado em 2020 jul 20]. Disponível em: http://cspace.eportuguese.org/tiki-list file gallery.php?galleryId=14.

18. Centro Latino-Americano e do Caribe de Informação em Ciências da Saúde. Capacitação no modelo da Biblioteca Virtual em Saúde no âmbito da rede ePORTUGUÊSe [Internet]. 2009 [citado em 2020 jul 20]. Disponível em: http://www.eventos.bvsalud.org/agendas/ eportuguese2009/.

19. Biblioteca Virtual em Saúde Brasil. Histórico [Internet]. 2010 [citado em 2018 ago 23]. Disponível em: http://brasil.bvs.br/vhl/sobre-a-bvs/historico-da-rede-bvs-no-brasil.

20. European Commission. Support to the development of Human Resource for Health. In: PALOP: European Community Contribution Agreement with an International Organization (9.ACP.MTR.004/1). Luanda; 2008. 